\title{
BMJ Open Dynamic LED-light versus static LED- light for depressed inpatients: study protocol for a randomised clinical study
}

\author{
Carlo Volf, ${ }_{1}^{1}$ Anne Sofie Aggestrup, ${ }^{1}$ Paul Michael Petersen, ${ }^{2}$ Carsten Dam-Hansen, ${ }^{2}$ \\ Ulla Knorr, ${ }^{1}$ Ema Erkocevic Petersen, ${ }^{3}$ Janus Engstrøm, ${ }^{4}$ Janus C Jakobsen, ${ }^{3}$ \\ Torben Skov Hansen, ${ }^{5}$ Helle Østergaard Madsen (D) , ${ }^{1}$ Ida Hageman, ${ }^{6}$ \\ Klaus Martiny (i) ${ }^{1}$
}

To cite: Volf C, Aggestrup AS, Petersen PM, et al. Dynamic LED-light versus static LEDlight for depressed inpatients: study protocol for a randomised clinical study. BMJ Open 2020;10:e032233. doi:10.1136/ bmjopen-2019-032233

- Prepublication history for this paper is available online To view these files, please visit the journal online (http://dx.doi. org/10.1136/bmjopen-2019032233).

Received 10 June 2019 Revised 19 December 2019 Accepted 07 January 2020

Check for updates

(c) Author(s) (or their employer(s)) 2020. Re-use permitted under CC BY-NC. No commercial re-use. See rights and permissions. Published by BMJ.

${ }^{1}$ Psychiatric Centre Copenhagen, University Hospital Copenhagen, Copenhagen, Denmark

${ }^{2}$ Department of Photonics Engineering, Technical University of Denmark, Lyngby, Denmark

${ }^{3}$ The Copenhagen Trial Unit, Centre for Clinical Intervention Research, Rigshospitalet, Copenhagen University Hospital, Copenhagen, Denmark

${ }^{4}$ Centre for Clinical Intervention Research, Rigshospitalet,

Kobenhavn, Denmark

${ }^{5}$ Chromaviso, Aarhus, Denmark

${ }^{6}$ Mental Health Services in the Capital Region of Denmark, Kobenhavn 0, Denmark

Correspondence to

Dr Klaus Martiny;

klaus.martiny@regionh.dk

\section{ABSTRACT}

Introduction Retrospective studies conducted in psychiatric inpatient wards have shown a relation between the intensity of daylight in patient rooms and the length of stay, pointing to an antidepressant effect of ambient lighting conditions. Light therapy has shown a promising antidepressant effect when administered from a light box. The emergence of light-emitting diode (LED) technology has made it possible to build luminaires into rooms and to dynamically mimic the spectral and temporal distribution of daylight. The objective of this study is to investigate the antidepressant efficacy of a newly developed dynamic LED-lighting system installed in an inpatient ward.

Methods and analysis In all, 150 inpatients with a major depressive episode, as part of either a major depressive disorder or as part of a bipolar disorder, will be included. The design is a two-arm 1:1 randomised study with a dynamic LED-lighting arm and a static LED-lighting arm, both as add-on to usual treatment in an inpatient psychiatric ward. The primary outcome is the baseline adjusted score on the 6-item Hamilton Depression Rating Scale at week 3 . The secondary outcomes are the mean score on the Suicidal Ideation Attributes Scale at week 3 , the mean score on the 17-item Hamilton Depression Rating Scale at week 3 and the mean score on the World Health Organisation Quality of Life-BREF (WHOQOL-BREF) at week 3 . The spectral distribution of daylight and LEDlight, with a specific focus on light mediated through the intrinsically photosensitive retinal ganglion cells, will be measured. Use of light luminaires will be logged. Assessors of Hamilton Depression Rating Scale scores and data analysts will be blinded for treatment allocation. The study was initiated in May 2019 and will end in December 2021.

Ethics and dissemination No ethical issues are expected. Results will be published in peer-reviewed journals, disseminated electronically and in print and presented at symposia.

Trial registration number NCT03821506; Pre-results.

\section{INTRODUCTION}

\section{Depression}

Depressive episodes, either as part of a major depressive disorder (MDD) or as part of a bipolar disorder (BD), are a prevalent and
Strengths and limitations of this study

- This is the first randomised clinical trial investigating the antidepressant effect of a dynamic light-emitting diode system in an inpatient psychiatric ward.

- The study has a rigorous design and a large sample size enabling it to find a clinically relevant antidepressant effect.

- Patient stay might not be of an adequate length to achieve full antidepressant effect of the lighting system.

- Light measurements will not accurately reflect corneal light exposure as wearable light sensors are not used.

leading cause of disability worldwide. Society suffers large direct and indirect losses due to lost work, sickness and early retirements, and WHO ranks major depression as a significant contributor to the global burden of disease. ${ }^{1}$ In Denmark alone (population of 5.8 million), the costs of depression amount to an estimated $€ 1.87$ billion per year. Depression can affect an individual to an extent that everyday activities and chores are insurmountable. These patients often have suicidal ideation and despair and need inpatient care including treatment with mood stabilisers, antipsychotics, antidepressants, psychoeducation, psychotherapy and occupational therapy. Typically, these patients will still suffer from varying degrees of depression when discharged ${ }^{23}$ and relapse, readmission and even suicide, on discharge, are major treatment challenges. ${ }^{4}$ Despite intense efforts during the last decades, there has been no breakthrough for either drug development or for any other treatment modality and $10 \%-25 \%$ of patients are treatment resistant. ${ }^{5}$ Thus, there is a need for the development of new treatment options. ${ }^{6}$ Light therapy has shown an antidepressant effect in outpatients, 
when using light box administered treatment, but studies in more severely depressed inpatients are warranted. ${ }^{7}$ The technological development has made it possible to some extent to mimic the temporal changes in intensity and spectral distribution of daylight with light-emitting diodes (LED) built into buildings. However, in this study we do not aim at creating actual daylight characteristics in the rooms but to provide a tailored spectral illuminance to enhance alertness, mood improvement and circadian regulation. This includes a focus on intrinsically photosensitive retinal ganglion cells (ipRGC)-influenced responses.

The present efficacy study thus investigates the possible antidepressant effect of a new, dynamic type of general room lighting in the treatment of a major depressive episode either as part of an MDD or a BD in patients admitted to an affective disorders ward.

\section{Bright light therapy}

Light has been used to treat medical conditions, such as melancholia or lethargy for at least a 1000 years $^{8}$ and daylight has been considered important for centuries when building hospitals. ${ }^{9}$ Animal research conducted through many decades has shown that dosage and timing of light have impact on reproduction, activity and sleep, ${ }^{10}$ and in the 1980s it was discovered that light could suppress melatonin secretion, in humans, ${ }^{11}$ indicating that light gives cues to the brain about night-time and season. The resultant clinical description of seasonal affective disorder and the theoretical analogy with hamster hibernation cycles led to the development of bright light treatment (BLT). ${ }^{12-15}$ It was later discovered that light pulses applied in the morning phase advance the sleep-wake cycle (and other rhythms), whereas light applied in the evening delays the sleep-wake cycle (and other rhythms). This is named the phase response curve (PRC) for light in humans, ${ }^{16}{ }^{17}$ and is the basis for the mechanism of 'entrainment' - the synchronisation of a self-sustaining oscillation (such as sleep) by an external forcing oscillation (such as daylight or artificially timed light). In 2000, a new non-visual retinal receptor, the ipRGC, was discovered in the human retina. ${ }^{18}$ The ipRGC receptors have a peak spectral sensitivity for the short-wavelength portion of the light spectrum $(460-480 \mathrm{~nm}) .{ }^{19}$ Since then it has been found, primarily through animal research, that the ipRGC regulates the circadian system through input to the suprachiasmatic nuclei and the pineal gland, but also, through newly found pathways, to brain structures known to be involved in depressive illness. ${ }^{20-23}$ This has given us a better understanding of how light can have a fast working antidepressant effect in humans and has stimulated research on the effect of using light with temporally shifting spectral compositions and intensity. The impact of the signals from the ipRGC is, among other effects, responsible for the ability of light to time and stabilise the sleep-wake cycle and to adjust the seasonal regulation of serotonin. ${ }^{24}$ LED-light can be tuned to be particularly rich in the short-wavelength spectrum in contrast to conventional compact fluorescent light. Thus, LED-light can be adjusted to maximise the impact on the ipRGC system. The temporal regulation of the spectral composition and intensity of LED-light is clinically important. Too much short-wavelength light in the evening will delay the sleep-wake cycle, through the PRC mechanism, causing a difficulty falling asleep (sleep-onset insomnia), ${ }^{25}$ whereas short-wavelength light in the morning will advance sleep and increase alertness. It should be noted that prereceptoral filters such as the lens tend to shift the spectral sensitivity of the melanopsin receptor to longer wavelengths.

There is an association between depression and late chronotypes $^{26}$ and late chronotypes is associated with non-response to treatment. ${ }^{27}$ Furthermore, patients with depression are prone to drift to later sleep schedules. ${ }^{3} \mathrm{We}$ should thus expect that dynamic lighting with enriched short-wavelength morning light and low content shortwavelength light in the evening would prevent drifting or even phase advance the sleep-wake cycle and thus augment the antidepressant treatment response. ${ }^{28}$

Since the publication of the first study on the effect of light therapy for depression by Rosenthal et al in $1984,{ }^{12}$ a large number of studies have been carried out to investigate the efficacy of BLT on both seasonal and non-seasonal depression. Light is one of the most thoroughly investigated chronotherapeutic treatments in addition to wake therapy ${ }^{29}$ and sleep phase advance. ${ }^{30} \mathrm{In}$ 2004, Tuunainen et al published the Cochrane systematic review 'Light therapy for non-seasonal depression'. ${ }^{31}$ The conclusion was that light therapy must be regarded as a promising treatment method, but because of the heterogeneity among the studies, methodological problems and a lack of systematic collection of adverse events (AEs), the recommendation of light therapy as a treatment of depression should be considered with some caution. In a systematic review from 2007, Even et al found an additive effect of light therapy when used as augmentation to antidepressant therapy in non-seasonal depression. ${ }^{32}$ In a systematic review from 2016, Perera et al included 20 randomised controlled trials using light therapy for nonseasonal depression and found an overall small antidepressant effect $(-0.41 ; 95 \%$ CI -0.64 to -0.18$)$, but with a high risk of bias and inconsistency between studies. ${ }^{33}$ In subgroup analyses (stand-alone light therapy vs adjunctive light therapy, morning light therapy vs evening light therapy or other times of day, light therapy for inpatients vs outpatients, placebo light conditions vs non-light-based placebo conditions), some support was found for a better effect of light when used as monotherapy, in the morning, for outpatients, and when compared with non-light-based placebos. Only 4 of the 20 studies had a low risk of bias on all items on the Cochrane Risk of Bias Tool. ${ }^{34-37}$ A Danish study found a significantly better effect of a combination of bright light therapy plus sertraline compared with dim red placebo light and sertraline, in 102 outpatients. ${ }^{34}$ In a Canadian study, 122 outpatients were compared in four groups: (a) active light plus active fluoxetine, (b) active light plus placebo fluoxetine, (c) inactive negative ion 
generator plus active fluoxetine, (d) inactive negative ion generator plus placebo fluoxetine. ${ }^{35}$ A significantly better effect was found in the group receiving the combination of active light plus active fluoxetine, as well as in the group receiving the combination of active light and placebo fluoxetine compared with the group receiving the combination of inactive negative ion generator plus placebo fluoxetine. A study performed in 84 elderly $(>60$ years) outpatients with non-seasonal depression found a statistically significant beneficial effect of active versus placebo light treatment. ${ }^{36}$ However, another study found no difference in depression outcome between groups, and a low reduction in depression severity across groups of $16 \%$, among 81 elderly outpatients (>60 years) with non-seasonal depression, treated with bright or placebo light administered at three different time-points. ${ }^{37} \mathrm{~A}$ more recent study showed significantly better effect of BLT compared with dim light treatment, when used as augmentation of mood stabilising medication, in 46 patients with $\mathrm{BD} .{ }^{38} \mathrm{~A}$ recent review showed that the risk of a switch from depression to mania, in patients with BD disorder treated with light therapy, was no higher than with antidepressant drug therapy alone. ${ }^{39}$

With conventional light therapy, patients are seated in front of a light box for 30-60 min in the morning. ${ }^{40}$ Even though it is technically possible to produce a tuneable light box, commercial light boxes often only deliver fixed spectral distributions. In treatment with dynamic LED-lighting, built-in luminaires can provide temporal changing intensity and spectral distribution and they substitute the general room-lighting. One of the main study design challenges with light therapy studies is the insufficient blinding of patients to light treatment conditions, and the lack of an appropriate control treatment (placebo). These challenges are not solved by using dynamic lighting as the change in colour and intensity is readily seen by the patients.

In conclusion, there is some evidence for the effect of traditional light box-administered treatment, primarily in seasonal and non-seasonal depression, less in $\mathrm{BD}$, but very little evidence for the effect of dynamic lighting. Therefore, a rigorously designed large study using dynamic lighting in patients with bipolar and unipolar major depression is warranted.

\section{Architecture, daylight and lighting in hospitals}

In Denmark (latitude $56^{\circ} \mathrm{N}$ ), daylight is scarce during the winter season ${ }^{41}$ and in addition, cold rainy weather tends to keep people inside. This reduces overall light exposure because indoor light intensities seldom exceed 100-300 lx, whereas outdoor light intensities often are higher than 2000-3000 lx, even on an overcast day. Light exposure might therefore not be adequate to entrain the sleep-wake cycle, and other circadian rhythms, or to sustain normal levels of alertness and mood. For counties with a northern location, the limited daylight during winter months make it pertinent to investigate how and if artificial LED-light can become an adjunct method to the treatment of depression in hospitals. There are casuistic observations of an association between onset of depression and sudden drop in solar irradiation ${ }^{42}$ and between measured light exposure and depression severity. ${ }^{43}$

Recent studies confirm ancient architectural principles about exposure to the morning sun, such as the late 19th century Nightingale pavilions facing southeast (SE) aiming to optimise exposure to the morning sun during winter darkness. ${ }^{44}$ Thus, these few studies have been carried out to investigate the effect of daylight or dynamic lighting on patients with depression. The first documented daylight experiment was done by Wirz-Justice $e t$ al in 1996 with seasonal patients showing a better antidepressant effect of a 1 hour walk outside compared with low-dose light therapy from a light box. ${ }^{45}$ Some studies have investigated the possible influence of light exposure on length of inpatient stay in psychiatric hospitals, retrospectively. In a sample of 174 inpatients treated for bipolar or unipolar major depression in Alberta, Canada $\left(53.6^{\circ} \mathrm{N}\right)$, Beauchemin and Hays found a length of stay of 16.9 days in bright rooms (east orientated) and 19.5 days in dimly lighted rooms (west-facing or indoor courtyard) $(\mathrm{p}<0.05) .{ }^{46}$ Benedetti et al investigated the length of inpatient stay for 187 patients with bipolar or unipolar depression in Milano, Italy $\left(45.5^{\circ} \mathrm{N}\right)$. For patients with $\mathrm{BD}$, the mean length of stay was 19.8 days in east-oriented rooms and 23.5 days in west-oriented rooms $(\mathrm{p}=0.02){ }^{47}$ No difference was found for patients with unipolar depression. In Berlin, Germany $\left(52.5^{\circ} \mathrm{N}\right)$, Staedt et al found a reduction in mean length of inpatient stay for patients with unipolar depression, from 25.91 (17.04) days to 22.04 (15.40) days ( $\mathrm{p}=0.023)$ when the psychiatric clinic was moved to new facilities equipped with shortwavelength enriched dynamic lighting system. ${ }^{48}$ However, when controlled for age the statistical significance was lost $(\mathrm{p}=0.083)$. In Mallorca, Spain $\left(39.7^{\circ} \mathrm{N}\right)$, Canellas $e t$ al found in a sample of 207 patients with depression as part of BD or MDD that the median inpatient stay was reduced from 14 days (IQR 8-19) to 11 days (IQR 6-15) $(p=0.007)$ when the ward was moved from a basement location to a new facility where the accumulated light exposure per day was $300 \%$ higher. ${ }^{49}$ In a study from our own group, we documented extreme midday differences in daylight exposure between hospital rooms facing SE and hospital rooms facing northwest (NW). Measured on a clear day, these differences were $57000 \mathrm{~lx}$ at the summer solstice, $38000 \mathrm{~lx}$ at the autumn equinox and $19000 \mathrm{~lx}$ at the winter solstice. We found a significantly shorter inpatient stay for patients staying in SE-facing room compared with NW-facing rooms. ${ }^{2}$ Latest, West $e t$ al examined the effect of dynamic light versus static light in a cluster randomisation design in two cerebral stroke rehabilitation units in Copenhagen, Denmark $\left(55.7^{\circ} \mathrm{N}\right)$. These patients were mostly bedridden, and the rooms were equipped with blinds that could regulate daylight. In the group randomised to dynamic light, patients had significantly lower mood scores (better), measured by the major depression inventory, compared with the group receiving 
static light. ${ }^{50}$ Moreover, patients had significantly elevated melatonin plasma levels at end point compared with baseline and evolved a significant rhythmicity of melatonin (cosinor analysis). ${ }^{51}$ There is also some evidence that dynamic lighting stabilises mood and improves sleep quality in individuals suffering from dementia. ${ }^{52-54}$

In summary, the evidence base for the effect of dynamic lighting in depression is still weak. There is only little knowledge on how LED-light should be implemented as well as the potentially beneficial or harmful effects of LED-light on patients with depression whether as part of a unipolar or bipolar disorder.

In hospitals, there will always be 'a dark side of the building' where facades will receive very little-if anymorning sun during wintertime. Dynamic LED-lighting enables us to tune the lighting conditions individually for each room in a building to compensate for insufficient daylight.

In the present randomised trial, we investigate the efficacy of a dynamic LED-lighting condition in inpatients with unipolar depression or BD. We have incorporated the results from our recent four single-room feasibility trial that focused on the performance and patient tolerability of the same dynamic LED system as used in this study. ${ }^{55}$

The present study will be an addition to the knowledge in this transdisciplinary field, combining medical science, architecture and engineering.

\section{Objective}

The objective of this study is to investigate the antidepressant effect of a newly developed dynamic LED-lighting system in an inpatient psychiatric ward. Patients with a current major depressive episode either as part of MDD or a $\mathrm{BD}$ will be randomised to receive either dynamic LED-lighting or static LED-lighting in combination with treatment as usual. We hypothesise that the group receiving dynamic LED-lighting will have a larger reduction in depression scores on the Hamilton Depression Rating Scale than the group receiving static LED-lighting and that the antidepressant effect of the intervention will be largest for individuals with BD.

\section{METHODS AND ANALYSIS \\ Design}

The study protocol is reported according to the Standard Protocol Items: Recommendations for Interventional Trials statement of randomised studies of non-pharmacological treatment. ${ }^{56}$ The study is a 3-week, randomised, controlled, single-blind, parallel-group study with a balanced allocation ratio (1:1) of adult patients diagnosed with depression either as part of MDD or BD. Patients will be randomly assigned to either dynamic LED-lighting or static LED-lighting with stratification for patients with a major depressive episode as part of a BD or MDD. We expect a ratio of 2:1 for these two subgroups. Participants will be psychometrically assessed at baseline and once a week for a total of 3 weeks. All other treatment elements at the ward will continue as usual. Patients who are discharged or transferred to another ward during the 3 weeks study period will be contacted for a follow-up assessment corresponding to the missing final assessment, to facilitate adherence to protocol.

\section{Study setting}

The study will be conducted at a specialised inpatient unit for affective disorders at the Mental Health Centre Copenhagen, located on the premises of Rigshospitalet. This ward delivers specialised treatment for patients with affective disorders (MDD, BD) consisting of psychoeducation, pharmacological treatment, electroconvulsive treatment, physiotherapy and occupational therapy performed by a transdisciplinary team of psychiatrists, nurses, physiotherapists and occupational therapists. The average period of admission is approximately 4-6 weeks. The ward has a capacity of 14 patients. The study intervention will include 10 single patient rooms each equipped with a lighting system that can provide either dynamic LED-lighting or static LED-lighting.

\section{Eligibility criteria}

All patients admitted to the inpatient ward will be considered for participation. Patients are considered eligible for inclusion into the study if they comply with the inclusion and exclusion criteria.

Inclusion criteria are: $>18$ years of age, a current major depressive episode as part of either MDD (Diagnostic and Statistical Manual of Mental Disorders (DSM)-IV) or BD (DSM-IV), patients with BD should be in current and recent (a minimum of 2 months before admission) mood stabilising treatment, informed consent and able to speak and understand Danish. Exclusion criteria are: severe suicidality corresponding to a score $>2$ on the Hamilton Depression Rating Scale item 3 or if the investigators are uncertain of the degree of suicidality, psychotic depressive features at time of inclusion or within the last 2 weeks, abuse of alcohol and/or drugs, a Young Mania Ratings Scale (YMRS) score of 7 or above or a current hypomanic or manic episode, coercive measures of any kind.

Discontinuation criteria are: serious adverse reactions (SARs), suspected unexpected serious adverse reactions (SUSARS), all listed exclusion criteria and if the patient wishes to leave the study. Intervention will be discontinued if one of the before-mentioned criteria are fulfilled but we will ask the patient for an endpoint assessment unless the patient wants to leave the study.

The eligibility criteria were chosen to represent a broad sample of the patient group to maximise the generalisability of the study results. Patients with severe suicidality is excluded because they are more often transferred to closed wards and thus lost to follow-up which reduces the quality of the study. The eligibility will be evaluated through case files and from interviews with the patients. The eligibility criteria are assessed from case records, from the Hamilton Depression Rating Scale, the YMRS 
and the Mini International Neuropsychiatric Interview (M.I.N.I.) instrument, which also contains a section on alcohol and drug abuse.

\section{Interventions}

In this study, the treatment with dynamic LED-lighting provides temporal change in intensity and spectral distribution throughout the 24-hour day. LED luminaires are built into the room and substitute the general room lighting. In this way, treatment is administered to the patient, throughout the day, when the patient is in the room, with a planned specified temporal and spectral distribution. This enables the system to provide brighter daytime lighting aiming at a phase advance of the circadian rhythms and to provide an alerting and moodenhancing effect. This is attempted by supplying more bright light rich in the short-waved region of the spectral range in the first part of the day, and warmer and less intense light with less short-wavelength light, later in the day and at night. This regulation should entrain and advance the sleep-wake cycle and thereby improve and stabilise mood. Due consideration has been taken as to not cause glare or other visual discomfort (guided from the results from our feasibility study). During evening and night, the light system provides low-intensity light with low short-wavelength content and thus with minimal impact on the circadian system to avoid sleep disturbances.

The intervention is used as a non-pharmacological add-on treatment. ${ }^{57}$ The dynamic luminaires are tuned to mimic the temporal intensity and spectral distribution of daylight, in a SE-facing room, with two sets of timingone for winter and one for summer. It is important to note that the temporal composition does not possess the daily variation as seen in nature, but it is defined as one reoccurring temporal composition for summer and one for winter.

Ten single rooms in the inpatient ward are equipped with the new LED-lighting system that can function either in static or dynamic mode in each room. The lighting system is operated from a control panel placed in a locked room at the ward. Patients who are admitted to single rooms and included in the study are randomly allocated to treatment with either dynamic LED-lighting or static LED-lighting.

\section{Dynamic LED-light group}

The dynamic LED-lighting consists of three lighting elements in each single patient room (A, B, C).

A: an LED panel built into the window jamb (in the vertical part of the window frame) mimicking the natural sunlight (see figure 1) as it is reflected in the window jamb from a white surface (RAL 9010).

This panel is turned on from 06:00 to 18:00 during the summer period (from 15 February to 31 October), and from 07:00 to 17:00 during the winter period (from 1 November to 14 February). The dimension of the panel is $1950 \times 310 \times 60 \mathrm{~mm}$. The light from this panel varies continuously in correlated colour temperature (CCT) from

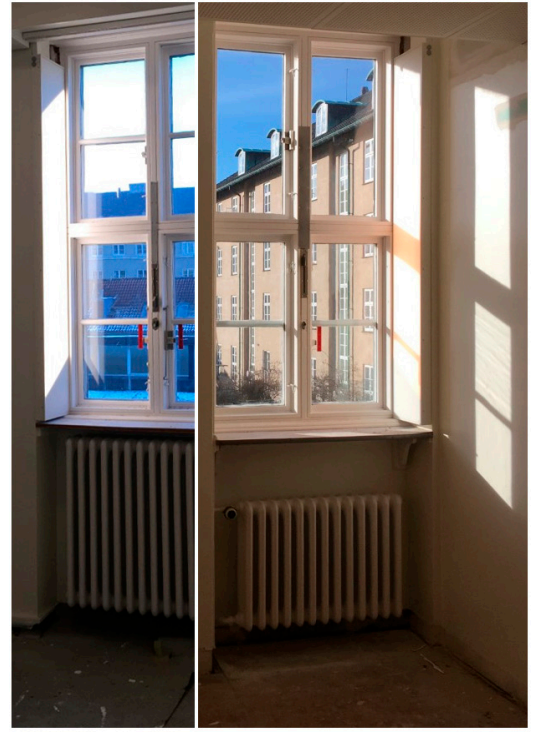

Figure 1 The left-hand picture shows how the light-emitting diode panel is mimicking sunlight reflections on the wall. The right-hand side of the picture shows natural sunlight reflexions on the wall.

$1800 \mathrm{~K} \mathrm{dim}$, warm-white light at 06:00 in the summer and 07:00 in the winter, rising to $5500 \mathrm{~K}$ bright white light from 9:00 to 14:00. From 14:00 and onwards, the light from the panel is reduced in both intensity and CCT, until fading out at $4000 \mathrm{~K}$ at 18:00 during the summer period and 17:00 during the winter period. This built-in LED panel cannot be switched off by the patients, but a curtain can be drawn to reduce intensity. The panel contains coolwhite (CW), warm-white (WW) and wide-spectrum amber (A) LEDs.

Daylight at dawn is rich in shortwave light, ${ }^{58}$ but we opted for a rather lower CCT at dawn (06:00/7:00) partly to mimic sunlight reflections in the window jamb on a white surface and because we believe, from clinical experience, that patients find it more calming to be woken up in light with a lower CCT. Patients in this study is expected to have high levels of anxiety and depression at awakening and a too high CCT might induce agitation.

B: two additional luminaires containing CW/WW/A LEDs are mounted in the ceiling with dynamic regulation of intensity and CCT during the whole 24-hour day. This light also varies from $1800 \mathrm{~K}$ dim, WW light to $5500 \mathrm{~K}$ at an intensity brighter than in a conventional patient room. The ceiling light can be turned off/on by the patient as preferred. During the summer, the dynamic LED-lighting is brightest between 09:00 and 14:00 and dimmest and warmest, from 23:00 to 06:00. In the winter period, the timings are changed to $09: 30$ to $13: 30$ and from 22:30 to 07:00 respectively.

C: a reading luminaire by the bed, with similar design and timing as the ceiling lighting and a regulation of CCT from 2100 to $5500 \mathrm{~K}$, with intensity kept relatively low, yet permitting reading while preventing too much suppression of melatonin in the evening. The reading luminaire 
can be turned off/on by the patient as preferred. The reading luminaire contains $\mathrm{CW} / \mathrm{WW} / \mathrm{A}$ LEDs.

All transitions are made as slow, imperceptible fades to mimic the nature of daylight through the daytime.

\section{Static LED-light group}

The static LED-light intervention uses the same luminaires as the dynamic LED-lighting intervention. In the static LED-light intervention, light output is completely static with regard to intensity, CCT and timing. The built-in window luminaire is permanently turned off and the ceiling luminaires (B) and reading luminaire (C) are both set to $3000 \mathrm{~K}$, at an intensity as expected in a typical patient room. Both ceiling and wall luminaires can be turned on/off as preferred by the patient. The use of the ceiling and reading luminaires will be logged continuously in both groups. In both groups there is a daylight contribution to the indoor illumination from windows.

\section{Light measurements}

The spectral irradiance from the LED-lighting systems was measured both horizontally and vertically in one of the patient rooms with blackout blinds in the window to exclude all daylight. The temporal distribution over a day, of the $\alpha$-opic irradiances (S-cone, M-cone, L-cone, rhodopic and melanopic) from the luminaires in the dynamic setting (A, B and C) and in the static setting (B and $\mathrm{C}$ ) were calculated horizontally from measurements $0.8 \mathrm{~m}$ above the floor ( $1 \mathrm{~m}$ from one corner of the room, $1 \mathrm{~m}$ out from the wall) just above the pillow of the patient bed using a Gigahertz Optik BTS-256-EF spectrometer ranging from 360 to $750 \mathrm{~nm}$ in $1 \mathrm{~nm}$ resolution. Vertical irradiances were measured simultaneously at $1.70 \mathrm{~m}$ above the floor on the wall adjacent to the pillow of the patient bed using a Konika Minolta CL-500A spectrometer ranging from 360 to 780 in $1 \mathrm{~nm}$ resolution. The $\alpha$-opic irradiances were calculated from the measured spectral irradiances (unit of $\mathrm{W} \mathrm{m}^{-2} \mathrm{~nm}^{-1}$ ). The $\alpha$-opic irradiances (unit of $\mathrm{W} \mathrm{m}^{-2}$ ) are the effective photobiological irradiances with the spectral irradiance spectrally weighed with the $\alpha$-opic action spectrum. ${ }^{59}$

The results from the measurements are shown in figure 2.

Figure 2A show results from the horizontal measurements with the dynamic setting shown in full lines and the static setting in dashed lines at specific times of the day and correspondingly figure $2 \mathrm{~B}$ shows results from the vertical measurements. During the study period, light sensors will measure real-time illuminance in all patient rooms. This illuminance sensor (Wireless Sensor Tag Pro ALS) will be placed on the wall beside the bed $170 \mathrm{~cm}$ above the floor and close to the head end of the bed. Spectral light measurements will only be recorded in one patient room for each geographical orientation (S, $\mathrm{E}, \mathrm{W}$ and $\mathrm{N}$ ) throughout the whole study period with a custom-built spectral sensor (AS7265X Multi Spectral Chipset, AMS) situated also on the wall beside the bed $170 \mathrm{~cm}$ above the floor and close to the head end of the bed. In the same four rooms, daylight will be measured with the same custom-built spectral sensor mounted in the windows facing outwards.

Figure 3 shows the measurement point from where the horizontal and vertical spectral measurement of the LEDlighting was done. In the same figure, the placement of the luminaires and the placement of the illuminance and spectral sensors are shown. Results of light measurements will be presented according to CIE S026. ${ }^{59}$

A suggestion on how to report light exposure in human clinical trials has been suggested in a recent paper by

B

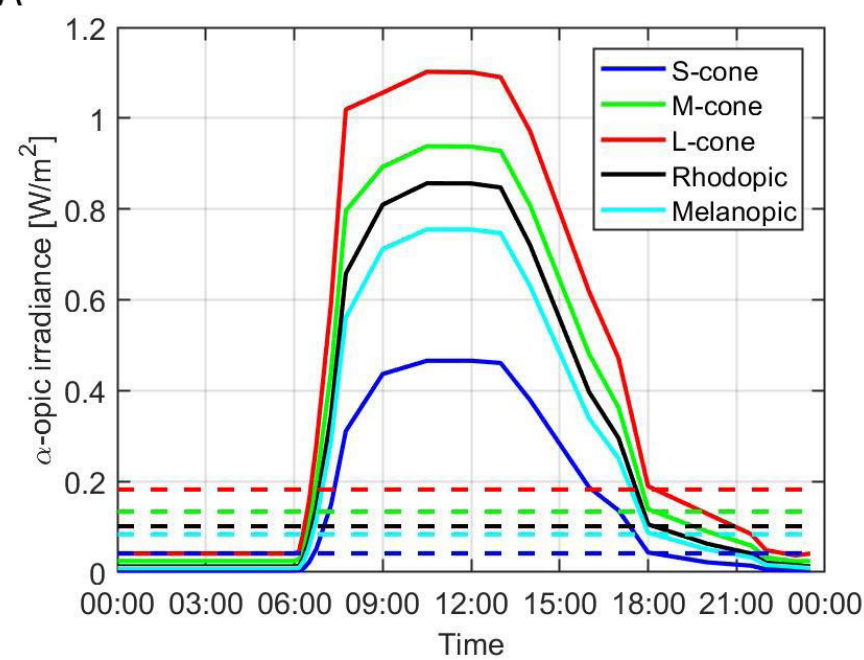

Figure 2 The temporal distribution of horizontal (A) and vertical (B) $\alpha$-opic irradiance of the light-emitting diode (LED)-lighting with daylight blacked out. Dynamic LED-light intervention is shown as solid lines and static LED-light intervention is shown as dashed lines. Measurement points and equipment are described in the 'Light measurement' section. The depicted timelines are for the summer intervention profile. Winter intervention profile differs in the way that the period between 06:00 and 18:00 during summer is contracted 1 hour in each end to 07:00 and 17:00 to enhance patient tolerability. 


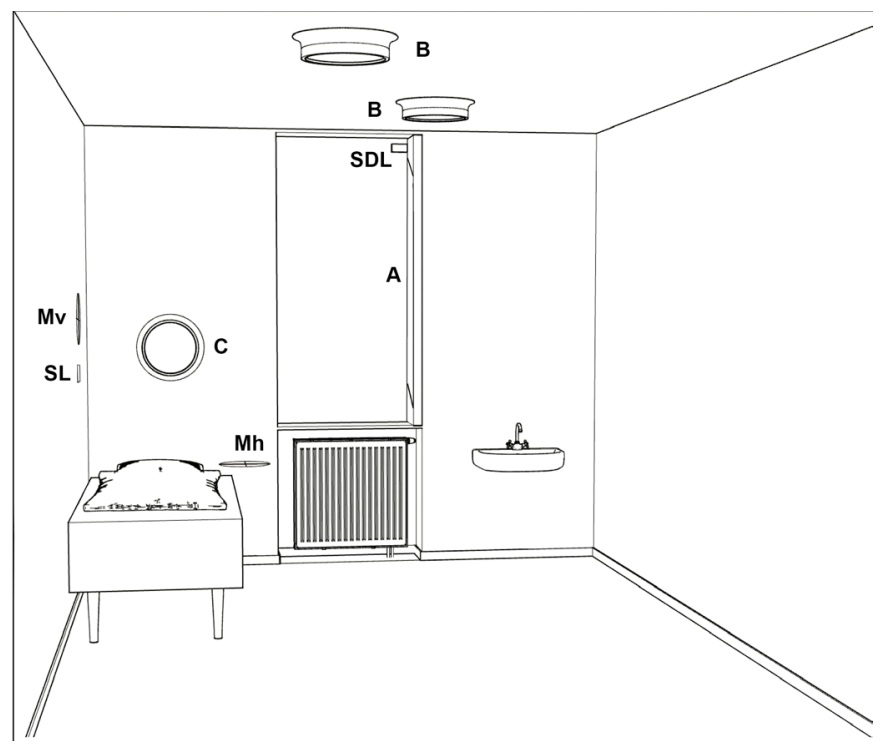

Figure 3 The placement of the luminaries where $A$ is the light-emitting diode (LED) panel built into the window jamb, $B$ is the two ceiling luminaires and $C$ is the reading luminaire. $\mathrm{Mv}$ and $\mathrm{Mh}$ indicates the measurement point for the vertical (Mv) and horizontal (Mh) spectral LED measurements performed in a patient room with blackout blind in the window to exclude daylight. SL indicates the permanent placement of the illuminance- and the spectral room sensors and SDL the permanent placement of the spectral daylight sensor.

Spitschan et $a l .{ }^{60}$ In this clinical trial, we will be able to report the $\alpha$-opic irradiances of the planned LED-lighting at referenced locations. Ideally, we should measure the light intensity and spectral distribution of the light that hits the cornea, but in this patient group we have decided not to use wearable light sensors due to reliability issues pertaining to the correct placement and use of such sensors.

\section{Concomitant care}

All participants will be offered the usual treatments at the ward. This includes nursing support to secure a daily structure, psychopharmacological drug therapy, electroconvulsive treatment, psychotherapeutic and psychoeducative methods, physiotherapeutic groups and occupational therapy. These concomitant treatments will have a foreseeable antidepressant effect that cannot be controlled for. However, this effect will be equally divided between the two groups due to the randomised design.

\section{Outcomes}

\section{Diagnostic measures}

The M.I.N.I. is used for diagnostic purposes by the investigators. This is a short, structured diagnostic interview based on the DSM-IV diagnostic system, and will be conducted to confirm depression diagnosis, assess comorbidity and any exclusion criteria for participation in the study. ${ }^{61}$ Investigators are certified in the use of the M.I.N.I. instrument.
Psychometrics and sociodemographics

Sociodemographic data are collected at baseline together with a treatment outcome expectancy rating. ${ }^{62}$ Psychometric assessments are performed at baseline and at the weekly evaluations. The rating window is set as the last 3 days for all instruments except for the Pittsburgh Sleep Quality Index (PSQI), ${ }^{63}$ which in this study covers the last 3 weeks and the YMRS which by tradition covers the last 7 days. ${ }^{64}$ The severity of depression is assessed by the Hamilton Depression Rating Scale, 17-item version $\left(\mathrm{HAM}_{17}\right) \mathrm{D}^{65}$ which includes the HAM$\mathrm{D}_{6}$ subscale, ${ }^{66}$ and by the Bech-Rafaelsen Melancholia Rating Scale focusing more on the cognitive symptoms of depression. ${ }^{67}$ The Hamilton rater is blinded to treatment allocation and is trained in the use of the HAM$\mathrm{D}_{17}$ by repeated group ratings with co-researchers. The YMRS is an 11-item, interviewer-administered scale, used to assess manic or hypomanic symptoms. It has a score range from 0 to 60 (highest severity of manic symptoms). ${ }^{64}$ The Suicidal Ideation Attributes Scale (SIDAS) is a 5 -item self-assessment scale with a score from 0 to 50 (highest level of suicidal thinking). ${ }^{68}$ The Udvalget for Kliniske Undersøgelser (UKU) scale (side-effect scale) is a generic side-effect scale covering degree of expected side effects from medications and supplemented in this study with items covering light sensitivity, all with scores from 0 to 3 (3 is severe side effect). ${ }^{69}$ Quality of life is assessed with the WHOQOL-BREF instrument measuring four life domains (physical health, psychological health, social relationships, environment), each with a score from 0 to 100 (best). ${ }^{70}$ The Morningness-Eveningness Questionnaire (MEQ) is a 19-item self-assessed questionnaire constructed to assess chronotype. A score below 42 indicates 'evening type' and a score above 58 indicates 'morning type'; and a score between 42 and 58 indicates 'intermediate type'. ${ }^{71}$ Sleep quality is assessed by the PSQI containing 11 self-reported items. These items are transformed into seven 'component scores', and a 'global score'. The components scores are 1) 'subjective sleep quality', 2) 'sleep latency', 3) 'sleep duration', 4) 'sleep efficiency', 5) 'sleep disturbances', 6) 'sleep medication' and 7) 'daytime dysfunction'. A 'global score' of 5 or above indicates poor sleep quality. ${ }^{63}$ Patients are asked to estimate their mean weekly sleep onset and offset, number of awakenings, sleep quality and duration and number of naps at each assessment. To evaluate the visual comfort in the room, we use a newly designed Visual Comfort Scale covering satisfaction and experience of the lighting conditions, covering the last week. ${ }^{72}$ To estimate the number of hours of exposure to the lighting condition, participants evaluate how much time they have spent in their room during the last week (room occupancy diary) in the time periods 06:00-12:00, 12:00-18:00 and 18:00-24:00. ${ }^{73}$ To estimate the exposure to daylight and to the built-in LEDpanel participants assess their use of curtains during the last week.

We believe that the participants will be able to fill in all the planned rating scales as many of these scales are quite 
short. However, we do offer to divide the inclusion visit on 2 days for patients with more pronounced cognitive impairment whether due to their illness or any sedating effect of psychotropic drugs.

It would have been valuable both to measure melatonin to assess circadian phase and of interest to use actimetry to get a more detailed information on sleep, but we have judged that these measures would be too complicated for participants to use together with the other procedures.

All events will be registered, and all serious adverse effects (SAEs), SUSARs and SARs will be reported to the ethical committee immediately.

We collect data on the use of medication at baseline and at week 3 from patient medical charts. Including the use of ad libitum medication.

\section{Ranking of outcomes}

The primary outcome is the baseline adjusted mean score on the HAM-D ${ }_{6}$ scale at week 3 .

The secondary outcomes are a) the mean score on the SIDAS scale at week $3, \mathrm{~b}$ ) the mean score on the HAM-D scale at week 3, c) the mean score on the WHOQOL-BREF at week 3. Exploratory outcomes are: a) the proportion of patients with one or more SAEs (according to ICHGCP 1997), b) the mean score on the visual comfort scale covering all 3 weeks, c) the mean reduction in $\mathrm{mg} /$ day from baseline to week 3 of zopiclone, d) the mean reduction in $\mathrm{mg}$ /day from baseline to week 3 of zolpidem, e) the mean reduction in $\mathrm{mg}$ /day from baseline to week 3 of quetiapine, e) the mean reduction in $\mathrm{mg} /$ day from baseline to week 3 of oxazepam, f) the mean score on the PSQI scale at week 3, g) the mean score on the MEQ scale at week $3, \mathrm{~h}$ ) the mean score on the HAM-D ${ }_{6}$ scale at 6 months.

A separate report will be made focusing on the total use of electrical energy for lighting in the static lighting group compared with the interventional LED-light group for a 1-year period.

\section{Participants timeline}

Enrolment and start of intervention are attempted to be within 5 days of admission to the ward. Assessments are performed at enrolment and once weekly for 3 weeks.

\section{Sample size and power estimations}

Sample size calculation was done using the SAS V.9.4 software using proc power for a two-sample independent sample t-test. In a previous study, ${ }^{2}$ on patients from the same ward, we found a mean HAM-D ${ }_{17}$ score at admission of 23. This corresponds to a HAM-D ${ }_{6}$ score of 13 . The number of participants has been estimated from an expected baseline-to-end point score reduction, on the HAM-D from $_{6} 13$ to 8 in the dynamic light group, and from 13 to 10 in the static light group. Hence, the minimal important difference is two points on the HAM$\mathrm{D}_{6}$ scale. With an expected SD of 3 , an $\alpha$ value of 0.05 and a power of $90 \%$, we will need a total of 98 patients for the primary outcome. However, to be able to perform explorative analyses we will aim at 150 patients.

Power estimations of secondary outcomes

1. SIDAS scale: we expect a SIDAS score of 20 at baseline with an SD of 8 and a reduction to a score of 5 in the dynamic light group and to 10 in the static light group. With an $\alpha$ value of 0.05 and a sample size of 98 participants, a power of 0.82 is estimated. With a sample size of 150 , power would be 0.95 .

2. $H A M-D_{17}$ scale: we expect a HAM-D ${ }_{17}$ score of 23 at baseline with an SD of 6 and a reduction to 14 in the active dynamic group and to 17.5 and the static light group. With an $\alpha$ value of 0.05 and a sample size of 98 participants, a power of 0.82 is estimated. With a sample size of 150 , power would be 0.95 .

3. WHOQOL-BREF questionnaire: we expect a WHOQOLBREF score of 20 at baseline with an SD of 17 and an increase to 45 in the dynamic light group and to 35 in the static light group. With an $\alpha$ value of 0.05 and a sample size of 98 participants, a power of 0.82 is estimated. With a sample size of 150 , power would be 0.95 .

\section{Recruitment}

The investigators will be in daily contact with the staff at the ward and will follow the flow of admissions. When a new patient is admitted and is occupying one of the 10 rooms with light equipment, the staff will give the patient an invitation to meet the investigator if no exclusion criteria are apparent. The patient will have the opportunity to read the participant information paper and the investigator will give a detailed oral information regarding the study. The oral information and written material will include information regarding the importance of obtaining outcome data in the case of drop-out due to early discharge and after 6 months. The patient will be asked to decide on participation within 2 days. If the patient accepts, the informed consent will be signed, and the inclusion and baseline assessments will begin. The patient record and the M.I.N.I. interview will be used to confirm the patient's eligibility. Included patients will be allocated to the next study number through the electronic case report form in the OpenClinica system, ${ }^{74}$ administered by the Copenhagen Study Unit (CTU). When submitted, the system randomises the participant to one of the two treatment groups: dynamic LED group or static LED group. This information is given to the patient. The estimated mean stay of patients is 1 month and with 10 light equipped room a total number of patients per year is 120 . With an expected inclusion rate of $50 \%$, we expect to finish last patient's last visit by June 2021.

\section{Allocation}

The randomisation will be web-based with a 1 (experimental intervention) to 1 (control intervention) allocation with a stratification for BD. A total of 150 patients will be randomised in the study. The primary investigator will enrol participants into the study. The randomisation is performed through the OpenClinica system, when 
participant's data have been entered in the electronic case report form (eCRF), and all inclusion criteria and no exclusion criteria are fulfilled.

\section{Blinding}

It is not possible to disguise the lighting condition to participants, primarily due to the extra light panel in the window that will be active in the dynamic lighting group. The depression outcome assessors will perform the assessment in a separate office in another department and will be blinded to treatment allocation. Participants are asked not to reveal their treatment allocation to the Hamilton assessor. Other investigators and data managers will not be blinded. The blinding will be broken in the event of a SAR or SUSAR.

Statistical analyses will be performed with the two intervention groups coded as 'A' and 'B' by two blinded statisticians employed at the CTU. The statisticians will independently and blindly analyse all data and present the results in two independent reports. A third investigator will compare these reports and discrepancies will be discussed. Both statistical reports will be published on CTU's website. Based on the statistical reports, two blinded conclusions will be drawn by the steering group: one assuming ' $\mathrm{A}$ ' is the experimental group and ' $\mathrm{B}$ ' is the control group-and one assuming the opposite. Based on these two blinded conclusions, two abstracts will be written and published on CTU's website. When the blinding is broken, the 'correct' abstract will be chosen and the conclusions in this abstract will not be revised.

\section{Data collection}

All assessments are carried out by psychometrically trained investigators (research nurse and senior consultant). The Hamilton ratings are conducted by a certified Hamilton rater blinded for group allocation. Hamilton certification is based on supervised group ratings with a difference of less than \pm two points on the HAM-D ${ }_{17}$ scale from the gold standard (most experienced rater). Study duration is 3 weeks, including baseline assessment (preintervention) and final assessment (postintervention). Patients who are transferred to another light-equipped room in the ward, during the 3-week period, can continue with their scheduled assessments and the lighting condition in the new room will be set according to the allocation.

\section{Data management}

Data will be collected through an eCRF developed by CTU in OpenClinica. Data in the eCRF are considered source data. The eCRF will use range checks for validation of entered data, and there will be an audit trail to monitor data entry. Data will be stored on servers locally at CTU with daily back-up. Data will also be collected from light sensors stored on independent hard drives. These data are also considered source data. The only pen-and-paper data are the informed consent and participants list. All data will be available to all authors.

\section{Statistical methods}

Continuous scale scores, including sleep scores, will be analysed in a linear regression model using available data from all included participants (intention to treat). The primary $\left(\mathrm{HAM}-\mathrm{D}_{6}\right)$ and secondary outcomes (SIDAS and HAM-D $_{17}$, and WHOQOL-BREF) will be entered in separate analyses as dependent variables in the linear regression model. Treatment group (static or dynamic), gender, length of actual depressive episode, bipolar/unipolar illness and baseline values of the dependent variable will be entered as explanatory variables. Results are given as mean, with confidence limits, SD and $\mathrm{p}$ values. A detailed statistical analysis plan will be published separately before the randomisation of the last participant. The significance level is set at 5\% two-tailed. Subgroup analyses will be performed for BD/MDD diagnosis. Missing data will be handled by multiple imputation techniques. ${ }^{75}$ No interim analyses are planned.

\section{Data monitoring and auditing}

According to Danish law, only drug studies are required to comply with Good Clinical Practice guidelines (GCP) ${ }^{76}$ We will, however, adhere to the GCP rules to secure study quality. Data analyses, performed by the primary investigator, will be supervised by a statistician. The study is subject to auditing from the regional ethical committee.

\section{Harm}

We do not expect that the study will expose participants to any serious hazard as their usual clinical management is maintained and the side-effect profile of bright light is low. ${ }^{34} 77$ All AEs will be recorded until the end of the follow-up period, and regulatory rules for reporting of SAEs and SARs will be adhered to. Any harm due to the study procedures is covered by the Danish Patient Compensation Association. The study will be stopped if there is a clinical suspicion of harm of the intervention, or if new evidence emerges that participants can come to harm due to the intervention.

\section{Ethics}

All participants will provide written informed consent before enrolment into the study. Informed consent will be obtained by the primary research investigator or a delegated study investigator. The study will be stopped if participants develop serious side effects. Patients can leave the study at any time at their own discretion and without any further effect on their continuous treatment at the ward. Any forthcoming protocol amendments will be submitted to the ethical committee and the Danish Data Protection Agency for approval. Written and oral information will be given at the ward, between the first and the fifth day of admission. The information will be given only by trained study investigators. The information will be given in an undisturbed office at the ward. Prior to the information visit, the patient will be informed of the opportunity to bring a friend or relative (third party). The 
patient will be given up to 2 days to consider participation before giving the informed consent.

Personnel at the ward will present information from patient records to permit investigators to determine any exclusion criteria. Patients eligible for enrolment will be asked by staff at the ward to participate in an information meeting. At inclusion, the patient will produce their civil registration number, name and sociodemographic data to the investigators. These data will be entered into the eCRF system and a participant-ID will be generated as part of the randomisation process. Participants' name, civil registration number and study number (from the eCRF) will be stored in a participant identification list in a secured data repository. Personal data in the eCRF will only be used for randomisation purposes, after which only the participant-ID will be used. At the end of the study, all paper material containing data will be transferred to a secure data repository. All study data will be handled according to the General Data Protection Regulation.

The final dataset will be accessible to all persons in the study group.

We do not expect any ethical issues. All regulatory rules will be followed, and the expected side effects of the dynamic lighting systems are rare and mild. We have taken precautions to find and deal with any emergent manic and suicidal symptoms.

\section{Dissemination}

Results will be published in peer-reviewed international journals, as posters and as oral presentations at international symposia. All data whether negative, positive or inconclusive will be reported in full. All members of the study group are coauthors with a pre-arranged order. No professional writers will be used. Depending on the journal of publication, part of the protocol, statistical code and dataset will be publicly available. All participants will be informed of the trial results.

\section{Acknowledgements The authors would like to thank Jais Elvekjær, New} Psychiatry Bispebjerg.

Contributors CV: design, planning, data acquisition, drafting and agreement of accountability for all aspects of the work. ASA: design, planning, data acquisition, drafting and agreement of accountability for all aspects of the work. TSH: planning, drafting and agreement of accountability for all aspects of the work. PMP: data acquisition, drafting and agreement of accountability for all aspects of the work. $\mathrm{CDH}$ : design, planning, drafting and agreement of accountability for all aspects of the work. UK: design, planning, drafting and agreement of accountability for all aspects of the work. EEP: planning, drafting and agreement of accountability for all aspects of the work. JE: planning, drafting and agreement of accountability for all aspects of the work. JJ: planning, drafting and agreement of accountability for all aspects of the work. HØM: planning, drafting and agreement of accountability for all aspects of the work. IH: planning, drafting and agreement of accountability for all aspects of the work. KM: design, planning, data acquisition, drafting and agreement of accountability for all aspects of the work. All authors read and approved the final manuscript.

Funding The following funded the study: Danish Energy/ELFORSK Foundation for Health Research, The Research Foundation of The Capital Region of Denmark, Merchant L. F. Foghts Foundation and The Toyota Foundation. None of the funding agencies has had any part in the design of the study or the writing of this manuscript.
Competing interests TSH is an employee of Chromaviso that has supplied the lighting system.

Patient consent for publication Patients were involved in the testing of tolerability of the lighting system. This influenced the setting of the dynamic lighting system. Patient also assisted in developing the Visual Comfort Scale that is used in the study to estimate tolerability of the LED-lighting system. Both involvements were done before the start of the study.

Ethics approval The study is approved by the Committee on Health Research Ethics of the Capital Region of Denmark with approval number H-19004525. The study is approved by the Danish Data Protection Agency with approval number VD-2018-515.

Provenance and peer review Not commissioned; externally peer reviewed.

Open access This is an open access article distributed in accordance with the Creative Commons Attribution Non Commercial (CC BY-NC 4.0) license, which permits others to distribute, remix, adapt, build upon this work non-commercially, and license their derivative works on different terms, provided the original work is properly cited, appropriate credit is given, any changes made indicated, and the use is non-commercial. See: http://creativecommons.org/licenses/by-nc/4.0/.

\section{ORCID iDs}

Helle Østergaard Madsen http://orcid.org/0000-0003-2000-8264

Klaus Martiny http://orcid.org/0000-0002-7317-5958

\section{REFERENCES}

1 Bromet E, Andrade LH, Hwang I, et al. Cross-National epidemiology of DSM-IV major depressive episode. BMC Med 2011;9:90.

2 Gbyl K, Østergaard Madsen H, Dunker Svendsen S, et al. Depressed patients hospitalized in Southeast-Facing rooms are discharged earlier than patients in Northwest-Facing rooms. Neuropsychobiology 2016;74:193-201.

3 Lauritsen L, Andersen L, Olsson E, et al. Usability, acceptability, and adherence to an electronic self-monitoring system in patients with major depression discharged from inpatient wards. $J$ Med Internet Res 2017;19:e123.

4 Bech P, Timmerby N, Martiny K, et al. Psychometric evaluation of the major depression inventory (MDI) as depression severity scale using the lead (longitudinal expert assessment of all data) as index of validity. BMC Psychiatry 2015;15:190.

5 Stimpson N, Agrawal N, Lewis G. Randomised controlled trials investigating pharmacological and psychological interventions for treatment-refractory depression. systematic review. $\mathrm{Br} J$ Psychiatry 2002;181:284-94.

6 Martiny K. Novel augmentation strategies in major depression. Dan Med J 2017;64.

7 Ravindran AV, Balneaves LG, Faulkner G, et al. Canadian network for mood and anxiety treatments (CANMAT) 2016 clinical guidelines for the management of adults with major depressive disorder: section 5. complementary and alternative medicine treatments. Can J Psychiatry 2016;61:576-87.

8 Wirz-Justice A, Benedetti F, Terman M. Chronotherapeutics for affective disorders: a clinician's manual for light and wake therapy. Basel: Karger, 2013.

9 LF M. On hospitals: their management, construction, and arrangements in relation to the successful treatment of disease, with remarks on the organisation of medical relief in the Metropolis. The Lancet 1881;117:979-82.

10 Foster RG, Kreitzman L. Rhythms of life : the biological clocks that control the daily lives of every living thing. London: Profile Books, 2004.

11 Lewy AJ, Wehr TA, Goodwin FK, et al. Light suppresses melatonin secretion in humans. Science 1980;210:1267-9.

12 Rosenthal NE, Sack DA, Gillin JC, et al. Seasonal affective disorder. A description of the syndrome and preliminary findings with light therapy. Arch Gen Psychiatry 1984;41:72-80.

13 Kripke DF, Mullaney DJ, Atkinson M, et al. Circadian rhythm disorders in manic-depressives. Biol Psychiatry 1978;13:335-51.

14 Kripke D. Photoperiodic mechanisms for depression and its treatment. Amsterdam: Elsevier/North Holland: Biological Psychiatry, 1981: 1249-52.

15 Arendt J. Melatonin and human rhythms. Chronobiol Int 2006;23:21-37.

16 Khalsa SBS, Jewett ME, Cajochen C, et al. A phase response curve to single bright light pulses in human subjects. J Physiol 2003;549:945-52. 
17 Czeisler CA, Duffy JF, Shanahan TL, et al. Stability, precision, and near-24-hour period of the human circadian pacemaker. Science 1999;284:2177-81.

18 Provencio I, Rodriguez IR, Jiang G, et al. A novel human opsin in the inner retina. J Neurosci 2000;20:600-5.

19 Münch M, Kawasaki A. Intrinsically photosensitive retinal ganglion cells: classification, function and clinical implications. Curr Opin Neurol 2013;26:45-51.

20 Hannibal J, Fahrenkrug J. Neuronal input pathways to the brain's biological clock and their functional significance. Adv Anat Embryol Cell Biol 2006;182:1-71.

21 Hattar S, Lucas RJ, Mrosovsky N, et al. Melanopsin and rod-cone photoreceptive systems account for all major accessory visual functions in mice. Nature 2003;424:75-81.

22 Do MTH, Yau K-W. Intrinsically photosensitive retinal ganglion cells. Physiol Rev 2010;90:1547-81.

23 Berman G, Muttuvelu D, Berman D, et al. Decreased retinal sensitivity in depressive disorder: a controlled study. Acta Psychiatr Scand 2018;137:231-40.

24 Lucas RJ, Peirson SN, Berson DM, et al. Measuring and using light in the melanopsin age. Trends Neurosci 2014;37:1-9.

25 Cajochen C, Frey S, Anders D, et al. Evening exposure to a light-emitting diodes (LED)-backlit computer screen affects circadian physiology and cognitive performance. J Appl Physio 2011:110:1432-8.

26 Chronotype NR. Depression and hippocampal volume: crosssectional associations from the UK Biobank. Chronobiology international 2019:1-8.

27 Chan JWY, Lam SP, Li SX, et al. Eveningness and insomnia: independent risk factors of nonremission in major depressive disorder. Sleep 2014;37:911-7.

28 Voderholzer U, Valerius G, Schaerer L, et al. Is the antidepressive effect of sleep deprivation stabilized by a three day phase advance of the sleep period? A pilot study. Eur Arch Psychiatry Clin Neurosci 2003;253:68-72

29 Martiny K, Refsgaard E, Lund V, et al. Maintained superiority of chronotherapeutics vs. exercise in a 20-week randomized follow-up trial in major depression. Acta Psychiatr Scand 2015;131:446-57.

30 Wirz-Justice A, Benedetti F. Perspectives in affective disorders: clocks and sleep. Eur J Neurosci 2019;6.

31 Tuunainen A, Kripke DF, Endo T, et al. Light therapy for nonseasonal depression. Cochrane Database of Systematic Reviews 2004;64:Cd004050.

32 Even C, Schröder CM, Friedman S, et al. Efficacy of light therapy in nonseasonal depression: a systematic review. J Affect Disord 2008;108:11-23.

33 Perera S, Eisen R, Bhatt M, et al. Light therapy for non-seasonal depression: systematic review and meta-analysis. BJPsych Open 2016;2:116-26.

34 Martiny K, Lunde M, Undén M, et al. Adjunctive bright light in nonseasonal major depression: results from clinician-rated depression scales. Acta Psychiatr Scand 2005;112:117-25.

35 Lam RW, Levitt AJ, Levitan RD, et al. Efficacy of bright light treatment, fluoxetine, and the combination in patients with Nonseasonal major depressive disorder: a randomized clinical trial. JAMA Psychiatry 2016;73:56-63.

36 Lieverse R, Van Someren EJ, Nielen MM, et al. Bright light treatment in elderly patients with nonseasonal major depressive disorder: a randomized placebo-controlled trial. Archives of general psychiatry 2011;68:61-70.

37 Loving RT, Kripke DF, Elliott JA, et al. Bright light treatment of depression for older adults [ISRCTN55452501]. BMC Psychiatry 2005;5:41.

38 Sit DK, McGowan J, Wiltrout C, et al. Adjunctive bright light therapy for bipolar depression: a randomized double-blind placebocontrolled trial. Am J Psychiatry 2018;175:131-9.

39 Benedetti F. Rate of switch from bipolar depression into mania after morning light therapy: a historical review. Psychiatry Res 2018;261:351-6.

40 Terman M, Terman JS. Light therapy for seasonal and nonseasonal depression: efficacy, protocol, safety, and side effects. CNS Spectr 2005;10:647-63.

41 Thieden E, Philipsen PA, Wulf HC. Ultraviolet radiation exposure pattern in winter compared with summer based on time-stamped personal dosimeter readings. Br J Dermatol 2006;154:133-8.

42 Summers L, Shur E. The relationship between onsets of depression and sudden drops in solar irradiation. Biol Psychiatry 1992;32:1164-72.

43 Oren DA, Moul DE, Schwartz PJ, et al. Exposure to ambient light in patients with winter seasonal affective disorder. The American journal of psychiatry 1994;151:591-3.
44 Nightingale F. Notes on hospitals. London: Longman, Green, Longman, Roberts, and Green, 1863.

45 Wirz-Justice A, Graw P, Kräuchi K, et al. 'Natural' light treatment of seasonal affective disorder. J Affect Disord 1996;37:109-20.

46 Beauchemin KM, Hays P. Sunny Hospital rooms expedite recovery from severe and refractory depressions. J Affect Disord 1996;40:49-51.

47 Benedetti F, Colombo C, Barbini B, et al. Morning sunlight reduces length of hospitalization in bipolar depression. J Affect Disord 2001;62:221-3.

48 Staedt J, Pless-Steinkamp C, Herfeld F, et al. Einfluss erhöhter Lichtintensität auf die Verweildauer von stationär behandelten depressiven Patienten. Nervenheilkunde 2009;28:223-6.

49 Canellas F, Mestre L, Belber M, et al. Increased daylight availability reduces length of hospitalisation in depressive patients. Eur Arch Psychiatry Clin Neurosci 2016;266:277-80.

50 West A, Simonsen SA, Zielinski A, et al. An exploratory investigation of the effect of naturalistic light on depression, anxiety, and cognitive outcomes in stroke patients during admission for rehabilitation: a randomized controlled trial. Journal of Alzheimer's disease : JAD 2019.

51 West AS, Sennels HP, Simonsen SA, et al. The effects of naturalistic light on diurnal plasma melatonin and serum cortisol levels in stroke patients during admission for rehabilitation: a randomized controlled trial. Int J Med Sci 2019;16:125-34.

52 Mishima K, Okawa M, Hishikawa Y, et al. Morning bright light therapy for sleep and behavior disorders in elderly patients with dementia. Acta Psychiatr Scand 1994;89:1-7.

53 Sloane PD, Williams CS, Mitchell CM, et al. High-Intensity environmental light in dementia: effect on sleep and activity. J Am Geriatr Soc 2007:55:1524-33.

54 Skjerve A, Holsten F, Aarsland DAG, et al. Improvement in behavioral symptoms and advance of activity acrophase after short-term bright light treatment in severe dementia. Psychiatry Clin Neurosci 2004;58:343-7.

55 https://pilotfeasibilitystudies.biomedcentral.com/articles/10.1186/ s40814-019-0548-9, 2020. Available: https://pilotfeasibilitystudies. biomedcentral.com/articles/ [Accessed 18-01-2020].

56 Chan A-W, Tetzlaff JM, Altman DG, et al. Spirit 2013 statement: defining standard protocol items for clinical trials. Ann Intern Med 2013;158:200-7.

57 Glickman G, Byrne B, Pineda C, et al. Light therapy for seasonal affective disorder with blue narrow-band light-emitting diodes (LEDs). Biol Psychiatry 2006;59:502-7.

58 Spitschan M, Aguirre GK, Brainard DH, et al. Variation of outdoor illumination as a function of solar elevation and light pollution. Sci Rep 2016;6:26756.

59 CIE. System for Metrology of optical radiation for ipRGC-Influenced responses to light, 2019. Available: https://www.techstreet.com/cie/ standards/cie-s-026-e-2018?product_id=2030705 [Accessed 24 Mar 2019].

60 Spitschan M, Stefani O, Blattner P, et al. How to report light exposure in human Chronobiology and sleep research experiments. Clocks Sleep 2019;1:280-9.

61 Sheehan DV, Lecrubier Y, Sheehan KH, et al. The Mini-International neuropsychiatric interview (M.I.N.I.): the development and validation of a structured diagnostic psychiatric interview for DSM-IV and ICD10. J Clin Psychiatry 1998;59 Suppl 20:22-33.

62 Martiny K, Refsgaard E, Lund V, et al. A 9-week randomized trial comparing a chronotherapeutic intervention (wake and light therapy) to exercise in major depressive disorder patients treated with duloxetine. J Clin Psychiatry 2012;73:1234-42.

63 Buysse DJ, Reynolds CF, Monk TH, et al. The Pittsburgh sleep quality index: a new instrument for psychiatric practice and research. Psychiatry Res 1989;28:193-213.

64 Lukasiewicz M, Gerard S, Besnard A, et al. Young mania rating scale: how to interpret the numbers? determination of a severity threshold and of the minimal clinically significant difference in the EMBLEM cohort. Int J Methods Psychiatr Res 2013;22:46-58.

65 Hamilton M. A rating scale for depression. Journal of Neurology, Neurosurgery \& Psychiatry 1960;23:56-62

66 Martiny K, Refsgaard E, Lund V, et al. The day-to-day acute effect of wake therapy in patients with major depression using the HAM-D6 as primary outcome measure: results from a randomised controlled trial. PLoS One 2013;8:e67264.

67 Bech P, Lauritzen L, Lunde M, et al. Psychometric analysis of the melancholia scale in trials with non-pharmacological augmentation of patients with therapy-resistant depression. Acta Neuropsychiatr 2014;26:155-60

68 van Spijker BAJ, Batterham PJ, Calear AL, et al. The suicidal ideation attributes scale (SIDAS): community-based validation study of a new 
scale for the measurement of suicidal ideation. Suicide Life Threat Behav 2014:44:408-19.

69 Lingjærde O, Ahlfors UG, Bech P, et al. The UKU side effect rating scale: a new comprehensive rating scale for psychotropic drugs and a cross-sectional study of side effects in neuroleptic-treated patients. Acta Psychiatr Scand 1987;76:1-100.

70 Skevington SM, Lotfy M, O'Connell KA, et al. The world Health organization's WHOQOL-BREF quality of life assessment: psychometric properties and results of the International field trial. A report from the WHOQOL group. Qual Life Res 2004;13:299-310.

71 Horne JA, Ostberg O. A self-assessment questionnaire to determine morningness-eveningness in human circadian rhythms. Int $J$ Chronobiol 1976;4:97-110.

72 NID-Group. A Visual Comfort Scale 2018 [New Interventions in Depression], 2018. Available: https://www.psykiatri-regionh.dk/nidgroup/assessment/Pages/default.aspx [Accessed 07 Jan 2019].
73 NID-Group. Room Occupancy Diary 2018 [New Interventions in Depression], 2018. Available: https://www.psykiatri-regionh.dk/nidgroup/assessment/Pages/default.aspx [Accessed 07 Jan 2019].

74 OpenClinica. Homepage for OpenClinica 2019, 2019. Available: https://www.openclinica.com/ [Accessed 10 Mar 2019].

75 Jakobsen JC, Gluud C, Wetterslev J, et al. When and how should multiple imputation be used for handling missing data in randomised clinical trials - a practical guide with flowcharts. BMC Med Res Methodol 2017;17:162-62.

76 LAEGEMIDDELSTYRELSEN. Homepage for Danish medicines Agency 2019, 2019. Available: https://laegemiddelstyrelsen.dk/en/ licensing/clinical-trials [Accessed 11 Mar 2019].

77 Brouwer A, Nguyen H-T, Snoek FJ, et al. Light therapy: is it safe for the eyes? Acta Psychiatr Scand 2017;136:534-48. 